\title{
Mining Popular Crime Patterns in Spatial Databases
}

\author{
B.V.S. Varma \\ CSE Department \\ Ideal Institute of Technology \\ Kakinada, Andhra Pradesh, India
}

\author{
V. Valli Kumari \\ CS\&SE Department \\ AU College of Engineering \\ Andhra University, Visakhapatnam, India
}

\begin{abstract}
Crime is one of the major threats to the society and is increasing rapidly in these days, so in order to control the crime rates many techniques and methods were brought into practise for the safety of the public. So such important task is given to the police by the data analysts. So, in this paper the proposing system mines popular crime patterns from spatial databases. Since the main part of the investigation will starts with the crime incident and the place of the crime. Therefore crime incident and place play a major role in mining process. This system helps in finding popular crime patterns speedy and results in better outputs.
\end{abstract}

\section{Keywords}

Frequent patterns, Popular patterns, Crime patterns, Crime Database, Spatial Database.

\section{INTRODUCTION}

Data mining is the process of extracting interesting patterns from large data. Applying the methods like neural networks, clustering, genetic algorithms (1950s), decision trees (1960s) and support vector machines (1980s) to data with the intention of extracting uncovered hidden patterns. These approaches are used by businesses, scientists and governments to transfer large volumes of data such as Airline Passenger Trip Records, Census data and Supermarket Scanner data to produce market research reports and documents. Data mining, "Extraction of hidden predictive information from large databases", is a powerful technology with great potentiality. The Data mining tools predict future trends and behaviours, allowing businesses to make proactive, knowledge-driven decisions and solutions. Usually large databases are searched for hidden patterns, finding predictive information that experts may miss because it lies outside their expectations and thoughts.

Crime is one of the global challenges facing humanity and requires persistent efforts in order to be curbed. The challenge of crime is still being tackled by security agencies, while crime analysts also persist in discovering trends in crime data. Many researchers are investigating various ways of tackling crime [1-6] and improving on existing algorithms used to derive crime patterns, in order to assist public safety and security agencies in achieving their objective of deterring crime and promoting citizen's safety. Recently there is a great movement in law enforcement towards more empirical. For any crime to be identified there is a need to have some sort of evidence and starting point of investigation. Police generally maintain a database of all the crimes that take place so in order for the future reference purpose. The main goal of data mining is to predict crime of understanding and analyzing the patterns in criminal behavior. The most frequent method to predict crime patterns involves the review of crime reports each day and those reports are compared with past crimes which is also time consuming process.

Spatial databases are used in real time applications such as urban planning, land surveying, geospatial web services as well as environmental assessments. Spatial database is a database that is used to store, query data and represents objects such as points, lines and polygons. Applying association rule mining on spatial database provides frequently occurring things in geographic area.

The rest of the paper is organized as follows. In section 2 related work is discussed. Section 3 the problem of popular crime patterns in spatial databases is defined. The process of mining popular crime patterns using PCrimespatial-growth algorithm and also describe how to draw the PCrimespatialtree structure for mining popular crime patterns in spatial databases in section 4. Our experimental results have been shown in section 5. Finally the paper has been concluded in section 6 .

\section{RELATED WORK}

Full and Partial Periodic Patterns are the pattern where every position in the pattern exhibits the periodicity. Periodic patterns in which one or more elements do not exhibit the periodicity are called partial periodic patterns. If $\{a\}\{b\}\{c\}\{b\}\{c\}\{b\}\{c\}\{a\}\{c\}\{d\}$ is an input sequence $\{b\}\{c\}$ is a full periodic pattern with period 2 . It is all also called as full periodic pattern because every position in the pattern exhibits the periodicity. Let a sequence $\{a\}\{b\}\{c\}\{a\}\{d\}\{c\}\{a\}\{c\}\{c\}$ contains a partial periodic pattern $\{a\}\left\{^{*}\right\}\{c\}$ with period 3 where the second element is not exhibiting the periodic behavior. In the study and analysis of data mining, the criminology can be categorized into crime control and crime suppression. Crime control tends to use knowledge from the analyzed data to control and prevent the occurrence of crime, whereas the criminal suppression tries to catch a criminal by using the history of a person recorded in databases. Brown et.al [7] developed an application framework called ReCAP (Regional Crime Analysis Program) for mining data in order to catch professional criminals using data fusion and data mining techniques. The Data fusion technique was used to manage, fuse and interprets information from various sources. The main purpose was to overcome confusion from conflicting reports and cluttered or noisy backgrounds. Data mining was used to automatically discover patterns and relationships in large databases. Crime detection and prevention techniques are applied to different applications ranging from cross-border security, Internet security to household crimes. Animesh Tripathy et. Al. [8] represents association rule mining for finding positive frequent objects and negative frequent objects.

J. Han et.al [9] proposed a frame work for mining periodic patterns from temporal databases. J Yang et.al [10] proposed a method to mine asynchronous periodic patterns in time series databases. K.Y. Haung et.al [11] also proposed a model to mine asynchronous periodic patterns in temporal databases.

De Bruin et. al. [12] introduced a framework for crime trends using a new distance measure for comparing all individuals based on their profiles and then clustering them accordingly. This method also provided a visual clustering of criminal 
careers and identification of classes of criminals. Abraham et al. [13] proposed a method to employ computer log files as history data to search some relationships by using the frequency occurrence of incidents. Then, they analyzed the result to produce profiles, which can be used to perceive the behavior of criminal. From the literature study, it could be concluded that crime data is increasing to very large quantities running into zeta bytes. This in turn is increasing the need for advanced and efficient techniques for analysis. Data mining as an analysis and knowledge discovery tool has immense potential for crime data analysis. As is the case with any other new technology, the requirement of such tool changes, which is further augmented by the new and advanced technologies used by the criminals. All these facts confirm that the field is not yet mature and needs further investigations. Spatial data gives the information about the location where longitude and latitude values say about a particular location.

\section{PROBLEM DEFINITION}

In this section the basic definitions of the problem are described related to popular crime patterns. Let item $=\left\{x_{1}, x_{2}\right.$, $\left.\mathrm{x}_{3}, \ldots \ldots \mathrm{x}_{\mathrm{n}}\right\}$ be a set of $\mathrm{m}$ domains items. A set of $\mathrm{n}$ transactions in transactional database are $\left\{\mathrm{t}_{1}, \mathrm{t}_{2}, \mathrm{t}_{3}, \ldots \ldots \mathrm{t}_{\mathrm{n}}\right\}$ where each transaction is a subset of each item in transactional database. $\left|t_{j}\right|$ is used $d_{j}$ to represent the length of transaction $t_{j}$. let $X=\left\{x_{1}, x_{2}, \ldots . x_{k}\right\}$ belongs to item be a pattern consisting of $\mathrm{k}$ items where $|\mathrm{x}|=\mathrm{k} \leq \mathrm{m}$. The projected database of $\mathrm{X}$ denoted as $\mathrm{DB}_{\mathrm{X}}$ is a set of TDB transaction that contain X. Here MTL $(X)$ and $\operatorname{STL}(X)$ are used to represent maximum length and total length of all transactions in $\mathrm{DB}_{\mathrm{X}}$ respectively.

Definition 1: the transaction popularity $\operatorname{Pop}\left(X, t_{j}\right)$ of a crime pattern $X$ in transaction $t_{j}$ measures the membership degree of $X$ in $t_{j}$. Then compute the membership degree based on the difference between the transaction length $\left|\mathrm{t}_{\mathrm{j}}\right|$ and pattern size $|x|$.

$$
\operatorname{Pop}\left(X, t_{j}\right)=\left|t_{j}\right|-|X|
$$

Definition 2: the long transaction popularity $\operatorname{Pop}\left(X, \mathrm{t}_{\mathrm{MTL}(\mathrm{x})}\right)$ of a crime pattern $X$ in transaction $t_{M T L(x)}$ measures the membership degree of $X$ in $t_{M T L}$, where $t_{M T L(x)}$ is the crime transaction having maximum length in $\mathrm{DB}_{\mathrm{X}}$.

$$
\operatorname{Pop}\left(X, t_{M T L(X)}\right)=\left(\max _{t j \in D B x}\left|t_{j}\right|\right)-|X|
$$

Definition 3: the popularity $\operatorname{Pop}(X)$ of a crime pattern $X$ in the TDB measures an aggregated membership degree of $X$ in all transaction in the TDB. It is defined as an average of all crime transaction popularities of $\mathrm{X}$.

$$
\operatorname{Pop}(X)=1 /\left|D B_{X}\right| \sum_{t_{j \in D B x}} \operatorname{Pop}\left(X, t_{j}\right)
$$

Definiton 4: A user specified minimum popularity threshold minpop is given, a crime pattern $\mathrm{X}$ is considered popular crime if its popularity is atleast minpop (i.e., $\operatorname{Pop}(\mathrm{X}) \geq$ minpop).

Definiton 5: the popularity $\operatorname{Pop}(X)$ of a pattern $X$ in the TDB measures an aggregate membership degree of $X$ in the TDB. It is defined in terms of $\operatorname{sumTL}(\mathrm{x})=\sum_{t j \in D B x}|\mathrm{tj}|$ as follows.

$$
\operatorname{Pop}(X)=\frac{1}{\left|D B_{X}\right|} \sum_{t_{j \in D B x}} \operatorname{Pop}\left(X, t_{j}\right)
$$

$$
\begin{gathered}
=\frac{1}{\left|D B_{X}\right|} \sum_{t_{j \in D B x}}\left(\left|t_{j}\right|-|X|\right) \\
=\frac{S T L(X)}{\left|D B_{X}\right|}-|X|
\end{gathered}
$$

The definition of $\operatorname{STL}(X)$ further confirms that the total transaction length $S T L(X)$ of $X$ satisfies the downward closure property (i.e., $S T L(X) \geq S T L\left(X_{-}\right)$if $\left.X \subseteq X^{\prime}\right)$.

\section{MINING POPULAR CRIME PATTERNS IN SPATIAL DATABASES}

This section describes the detailed structure, functioning, underlying principles and applications of the PCrimespatialtree approach to crime mining, using some illustrative analogies. The proposed advancement to the traditional frequent pattern growth (TFP-Growth) model is described here. This involves using a descriptive statistical approach, as a strategy for the pruning phase in the TFP-Growth model, and adopting a pattern based paradigm to identify subtle crime patterns.

Consider as a typical crime database $($ Crime $D B)$ with categorical crime attributes, which comprises incident location, suspect and victim information, day of the week, time and date, weapons used, and crime scene status, to mention a few. The first step is to manage and identify the attributes of interest that are available in the crime investigation report (Table 1).

Table 1. Crime Database (CrimeDB)

\begin{tabular}{clcccccc}
\hline Date & Time & \multicolumn{2}{c}{ Location } & \multicolumn{3}{c}{ Type of crime No. of people involved } \\
& & Place & long & lati & & \\
& & & & & & 4 \\
\hline $18-08-2004$ & $6: 30 \mathrm{AM}$ & Gnt & -149.007 & 63.809 & kidnap, rape & 2 \\
$20-03-2005$ & $8: 30 \mathrm{PM}$ & Vij & -148.897 & 63.766 & rape, murder, robbery & 2 \\
$14-02-2010$ & $7: 00 \mathrm{PM}$ & Vij & -148.967 & 63.824 & kidnap, murder, robbery, burglary & 3 \\
$08-06-2012$ & $1: 30 \mathrm{PM}$ & Gnt & -149.026 & 63.803 & kidnap, robbery, burglary & 2 \\
$12-05-2013$ & $4: 00 \mathrm{PM}$ & Tni & -149.046 & 63.795 & rape, murder, burglary & 2 \\
$08-032014$ & $12: 30 \mathrm{PM}$ & Tni & -136.231 & 62.141 & Kidnap, rape, murder, robbery & 4
\end{tabular}

To illustrate how CrimeTDB-Growth mines crime data, Let consider a simple analogy form. Suppose a query (involving location information and corresponding crime incidents that occurred there) passed to obtain the crime transaction database (CrimeTDB) which is further encoded or transformed with Crime Transaction Identification (CT ID) being the unique identifier for each crime transaction and Crime Incident Information (CII). Each crime incident and location type has been encoded with abbreviations, in order to reduce processing time. CrimeTDB over CrimeDB is a set of transactions projected over Crime $D B$. Now, mine popular crime patterns with regard to location.

Thus all crime item-sets $X$, whose support value is less than the specified minimum support threshold, say $\lambda$, are considered unpopular and discarded as seen in [14]. The remaining crime attributes values are considered popular and arranged in support descending order as presented in the second half. Thus, discarding unpopular items in eventually results in CrimeTDB presented. This heuristic (of ordering by decreasing support value) is typically used because the size of 
the FP-Tree will depend on how the crime items are ordered. Thus common prefixes can be shared during the PCrimespatial-tree construction.

The PCrimespatial-Growth algorithm typically consists of two major steps; building the PCrimespatial-tree and extracting popular item-sets from the PCrimespatial-tree. The PCrimespatial-tree is usually compact and smaller than the uncompressed data (since many crime transactions typically share prefixes or crime items). Thus the worst case scenario would be when every transaction has a unique set of crime items (none in common), while the best case scenario would be when all transactions contain the same set of crime items.. Building the PCrimespatial-tree consists of two major passes, which involves the following:

\section{1) First Pass}

- Scan the CrimeTDB and find support for each crime item.

-Pruning Phase: discard unpopular crime items (items whose support is less than that Sort popular crime items) in decreasing order of support count.

\section{2) Second Pass}

- Construct the PCrimespatial-tree from the pruned CrimeDB. - Generate popular crime item-sets.

To create a root node (null) and read crime transaction CT1:

Table 2. CrimeTDB

\begin{tabular}{cccccc}
\hline Date & Time & \multicolumn{3}{c}{ Location } & \multicolumn{1}{c}{ Type Of Crime } \\
& & Place & Longitude & Latitude & \\
\hline & & & & & \\
$18-08-2004$ & $6: 30 \mathrm{AM}$ & Gnt & -149.007 & 63.809 & kidnap, rape \\
$20-03-2005$ & $8: 30 \mathrm{PM}$ & $\mathrm{Vij}$ & -148.897 & 63.766 & rape, murder, robbery \\
$14-02-2010$ & $7: 00 \mathrm{PM}$ & $\mathrm{Vij}$ & -148.967 & 63.824 & kidnap, murder, robbery, burglary \\
$08-06-2012$ & $1: 30 \mathrm{PM}$ & Gnt & -149.026 & 63.803 & kidnap,robbery,burglary \\
$12-05-2013$ & $4: 00 \mathrm{PM}$ & Tni & -149.046 & 63.795 & rape,murder,burglary \\
$08-032014$ & $12: 30 \mathrm{PM}$ & Tni & -136.231 & 62.141 & Kidnap,rape, murder,robbery \\
\hline
\end{tabular}

$\{\mathrm{kp}, \mathrm{rp}\}$, then create two nodes $k p$ and $r p$ and map it to a path, setting counts of $k p$ and $r p$ to 1 . Read CT2 : $\{\mathrm{rp}, \mathrm{md}, \mathrm{rb}\}$ likewise and map it to another path, but add the link between the "rp's". Note that CT1 and CT2 have disjoint paths, since they do not have a common prefix, even though both contain $r$ $p$. This process is repeated for every $C T_{i}, i \in\{1,2, \ldots, 10\}$ Table 3 , in order to generate the PCrimespatial-tree. Use a fixed order approach so that paths can overlap when crime transactions share crime items (i.e., have identical prefix) at any given time, in which case counters are incremented accordingly. PCrimespatial-tree has a higher compression rate when more paths overlap. Thus, a compact representation will be the result.

After CrimeTDB-Tree construction, it becomes easy to construct the conditional pattern base that can mine all popular item-sets present. The limitation with popular is that it may not fit into the memory when the crime transaction set becomes very large. Hence, the algorithm is improved upon in several ways.

The main asset of the system is the spatial information that is required for finding out the exact location of the crime. Spatial information is nothing but the longitude and the latitude values of a particular location which is represented in the form of $\{x, y\}$-axis, $y$-axis. Table 2 gives the information required for finding the popular patterns, so to make the work simpler, the crime incidents are encoded as shown in the Table 2.
During the process of mining the popular crime patterns, the calculation of popularity satisfies the downward closure property i.e if one item is considered as unpopular then its superset is also considered as unpopular which helps in reducing the search space by deleting the unpopular patterns and thus the process of mining will be faster. Sometimes the popularity does not satisfy downward closure property, therefore the readers took the mining of popular crime patterns as a challenging step.

\section{Construction of PCrimespatial-tree}

To mine popular patterns, the proposed PCrimespatial- growth algorithm, consists of two key procedures: (i) construction of a PCrimespatial-tree and (ii) mining of popular crime patterns from the PCrimespatial-tree.

First build a tree structure - called CrimeTDB-tree to capture the necessary information from the CrimeDB with only two scans of the CrimeDB. Recall that $\operatorname{Pop}(X)$ does not satisfy the downward closure property. So, unpopular items need to be kept in the CrimeTDB-tree as some of their supersets may be popular. Fortunately, recall from that $\operatorname{STL}(X)$ satisfies the downward closure property. So, not all unpopular items need to be kept. Some of them can be pruned.

Table 3. CrimeTDB (crime incidents)

\begin{tabular}{ll}
\hline TID & CRIME INCIDENTS \\
\hline CT1 & $\{\mathrm{kp}, \mathrm{rp}\}$ \\
CT2 & $\{\mathrm{rp}, \mathrm{md}, \mathrm{rb}\}$ \\
CT3 & $\{\mathrm{kp}, \mathrm{md}, \mathrm{rb}, \mathrm{bg}\}$ \\
CT4 & $\{\mathrm{kp}, \mathrm{rb}, \mathrm{bg}\}$ \\
CT5 & $\{\mathrm{rp}, \mathrm{md}, \mathrm{bg}\}$ \\
CT6 & $\{\mathrm{kp}, \mathrm{rp}, \mathrm{md}, \mathrm{rb}\}$ \\
CT7 & $\{\mathrm{kp}, \mathrm{sl}, \mathrm{ar}\}$ \\
CT8 & $\{\mathrm{kp}, \mathrm{rp}, \mathrm{rb}\}$ \\
CT9 & $\{\mathrm{kp}, \mathrm{rp}, \mathrm{rb}\}$ \\
CT10 & $\{\mathrm{kp}, \mathrm{rp}, \mathrm{md}\}$
\end{tabular}

To construct a CrimeTDB-tree, scan the CrimeTDB to find the $\operatorname{support}(x)$, maximum transaction length $\operatorname{MTL}(\mathrm{X})$ and the popularity $\operatorname{CrimeTDB}(x)$ for each singleton $x$ in the CDB. Then, perform the super-pattern popularity check and safely delete a pattern $x$ if PopularUB $(x)<$ minpop (where $x$ is an extension of $x$ ). Then scan the CrimeTDB the second time to insert each transaction into the CrimeTDB-tree in a similar fashion as the insertion process of FP-tree.

Let us show how to construct a PCrimespatial-tree for the CrimeTDB shown in Table 1 with minpop $=2.2$ With the first database scan, it obtains the following information in the form of <x: $\operatorname{support}(x), \operatorname{MTL}(x), \operatorname{Pop}(x)>\quad$ for each of the $m=10$ domain items, i.e., getting the following values after the scan. Let us assume the min-pop value min-pop $<=2.2$

$\langle$ kp: $8,4,2.125\rangle,\langle$ rp: 7, 4, 2〉, ,<md: 6, 4, 2.33>, <rb: 5, 4, $2.4>,\langle$ bg: 3, 4, $2.33>$, <sl: 1, 3, 2>, <ar: 1, 3, 2>

Although kp, rp, sl, ar are unpopular because of the min-pop value being 2.2 but consider them completely as the unpopular crime pattern because of the reason that their super patterns may be popular. So for that reason there is a need to perform the super pattern popularity check. 
The popularity upper bounds of $\mathrm{kp}, \mathrm{rp}, \mathrm{sl}$, ar are

$$
\begin{aligned}
& \mathrm{kp}=8-2=6 \\
& \mathrm{rp}=7-2=5 \\
& \mathrm{sl}=3-2=1 \\
& \mathrm{ar}=3-2=1
\end{aligned}
$$

As the values of $K p \& R p$ are greater than the min-pop value considered i.e 2.2 therefore don't delete the crime incidents from the database.

But the case of the other two crime incidents is not like that because their min-pop values are less than the values that is taken for verification. Since the crime incidents $\mathrm{Sl}, \mathrm{Ar}$ are not satisfying the super popularity check so they can be deleted completely i.e they are not eligible for the popular pattern check.

Now scan the PCrimespatial-tree the second time. Compute the length of each transaction, remove all items that are not in the H-table, and sort the remaining items in each transaction according to the H-table order. Fig. 1(a) shows the contents of the H-table $(x: \operatorname{support}(x), \operatorname{STL}(\mathrm{X}), \operatorname{MTL}(\mathrm{X}))$ and the popular tree structure after inserting $t 1$ of PCrimespatial-tree.

\section{H-TABLE}

\begin{tabular}{|c|}
\hline Kp: $8,25,4$ \\
Rp:7,21,4 \\
Md:6,16,4 \\
Rb:5,17,4 \\
Bg:3,10,4
\end{tabular}

Sharing a common prefix for different transactions the PCrimespatial-tree allows to extract popular crime pattern of common prefix that to be compact. One can observe that a PCrimespatial-tree is a highly compact tree structure. To mine popular crime patterns, the PCrimeSpatial-growth algorithm applies two key procedures: (i) construction of a PCrimespatial-tree and (ii) mining of popular crime patterns from the PCrimespatial-tree. The PCrimespatial-growth finds popular patterns from the PCrimespatial-tree.

Finding individual popularity of each crime incident from equation

Popularity of $\mathrm{Kp}=(|\mathrm{t} 1|+|\mathrm{t} 3|+|\mathrm{t} 4|+|\mathrm{t} 6|+|\mathrm{t} 7|+|\mathrm{t} 8|+|\mathrm{t} 9|+|\mathrm{t} 10|) /$

$|\mathrm{t} 1, \mathrm{t} 3, \mathrm{t} 4, \mathrm{t} 6, \mathrm{t} 7, \mathrm{t} 8, \mathrm{t} 9, \mathrm{t} 10|-|\mathrm{kp}|$

$=2+4+3+4+3+3+3+3 / 8-1$

$=2.125$

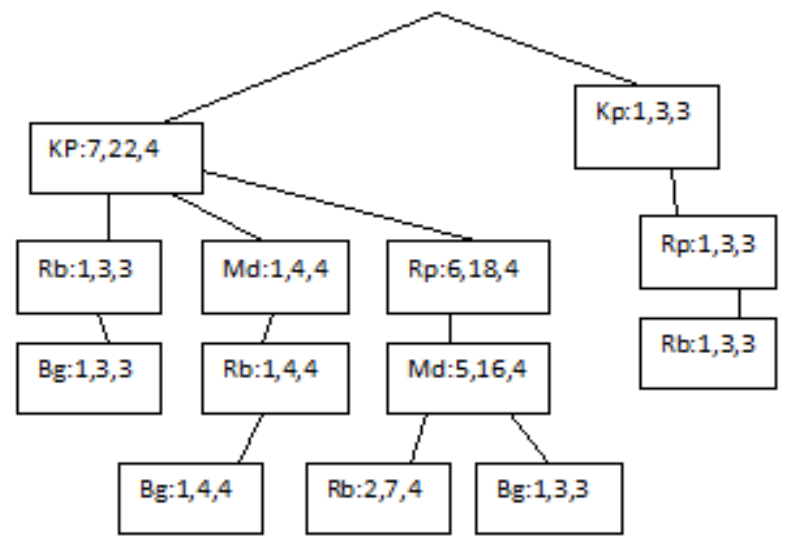

Fig 1.PCrimespatial-tree

Similarly find the popularity of other crime incidents also

Like

Popularity of $\mathrm{Rp}=|\mathrm{t} 1|+|\mathrm{t} 2|+|\mathrm{t} 5|+|\mathrm{t} 6|+|\mathrm{t} 8|+|\mathrm{t} 9|+|\mathrm{t} 10| / /$

$$
\begin{aligned}
& |\mathrm{t} 1, \mathrm{t} 2, \mathrm{t} 5 . \mathrm{t} 6, \mathrm{t} 8, \mathrm{t} 9, \mathrm{t} 10|-1 \\
& =2
\end{aligned}
$$

Popularity of $\mathrm{Md}=|\mathrm{t} 2|+|\mathrm{t} 3|+|\mathrm{t} 5|+|\mathrm{t} 6|+|\mathrm{t} 8|+|\mathrm{t} 10| /$

$$
\begin{aligned}
& |\mathrm{t} 2, \mathrm{t} 3, \mathrm{t} 5 . \mathrm{t} 6, \mathrm{t} 8, \mathrm{t} 10|-1 \\
& =2.33
\end{aligned}
$$

Popularity of $\mathrm{Rb}=|\mathrm{t} 2|+|\mathrm{t} 3|+|\mathrm{t} 4|+|\mathrm{t} 6|+|\mathrm{t} 9| /$

$$
\begin{aligned}
& |\mathrm{t} 2, \mathrm{t} 3, \mathrm{t} 4, \mathrm{t} 6, \mathrm{t} 9|-1 \\
& =2.4
\end{aligned}
$$

Popularity of $\mathrm{Bg}=|\mathrm{t} 3|+|| \mathrm{t} 4|+| \mathrm{t} 5 \mid /$

$$
\begin{aligned}
& |\mathrm{t} 3, \mathrm{t} 4, \mathrm{t} 5|-1 \\
& =2.33
\end{aligned}
$$

Popularity of $\mathrm{Sl}=|\mathrm{t} 7| / \mathrm{t} 7-1=2$

Popularity of ar $=|\mathrm{t} 7| / \mathrm{t} 7-1=2$

The popularity of each crime is calculated above and the minpop is 2.2. The length-1 popular crime patterns are greater than or equal to 2.2. The process continues for length-2 popular crime patterns and so on until no popular crime patterns found.

\section{EXPERIMENT RESULTS}

\section{Compactness of the Pop-Tree}

The compactness of a PCrimespatial-tree existed in terms of number of Pop-tree nodes. Consequently, every item in the dataset passes the lazy pruning phase and contributes to the tree. Hence, for a particular portion of the dataset, the tree size (i.e., number of nodes) is the same with the variation of minpop. The size of the tree structure gradually reduced with the increase of minpop. As expected, in both datasets, the number of nodes increased with the increase in size of database. However, a Pop-tree structure is compact enough to fit into a reasonable amount of memory.

\section{Scalability of Pop-Growth}

Clearly, as the minpop decreases, the overall tree construction time,mining time and required memory increase. However, the PCrimeSpatial-tree shows a stable performance with a linear increase in runtime and memory consumption as the 
minpop decreased for the dataset. Moreover, the results demonstrate that, the PCrimeSpatial -tree can mine the set of popular patterns on this dataset for a reasonably small value of popularity threshold with a considerable amount of execution time and memory. Furthermore, the PCrimeSpatial-tree structure and the PCrimeSpatial-growth algorithm are scalable for popularity threshold values and memory.

\section{Runtime}

The execution time that the PCrimespatial-growth requires forming popular patterns and changes in minpop. The execution time includes all the steps of $\mathrm{H}$-table construction, the Pop-tree building and the corresponding mining. As the database size increased and minpop decreased, the tree structure size and number of popular patterns increased. Hence, comparatively longer time was required to generate large number of popular patterns from large trees.

\section{Reduction on the Number of Patterns When Changing minpop}

The number of patterns generated by PCrimespatial-growth algorithm varies when data set size and minpop varies. Depending on dataset characteristics, the reduction rate varied. It is to note that the pattern count reduction rate was very similar irrespective of its different size. It also observed that the pattern generation characteristics of the proposed popular pattern mining algorithm were consistent with the variation of minpop and database size.

The experiments are done on the crime datasets which are available as open source in National Crime Records Bureau, India http://data.gov.in, and few datasets of UK and USA are also considered http://data.gov.uk/ /crime_statistics, http://data. vancouver/ details.htm. Some other datasets like city of Chicago crime datasets from the year 2001 to present, it contains 58,71,658 number of crime records with 20 different attributes.

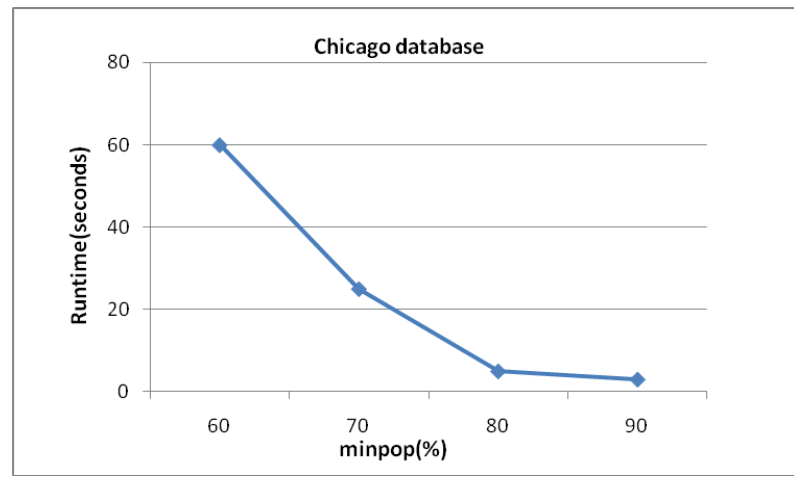

Fig 1: Exection time over chicago crime databse

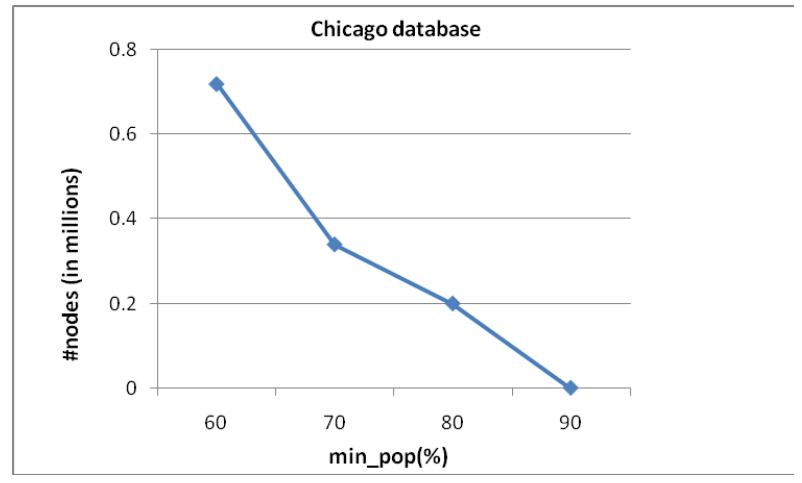

Fig 2: Memory utilization for Chicago database
Figure 1 represents execution time over Chicago crime database, where popularity increases mining time also increases propotionally. Figure 2 represents memory usage for popular crime patterns over Chicago crime database.

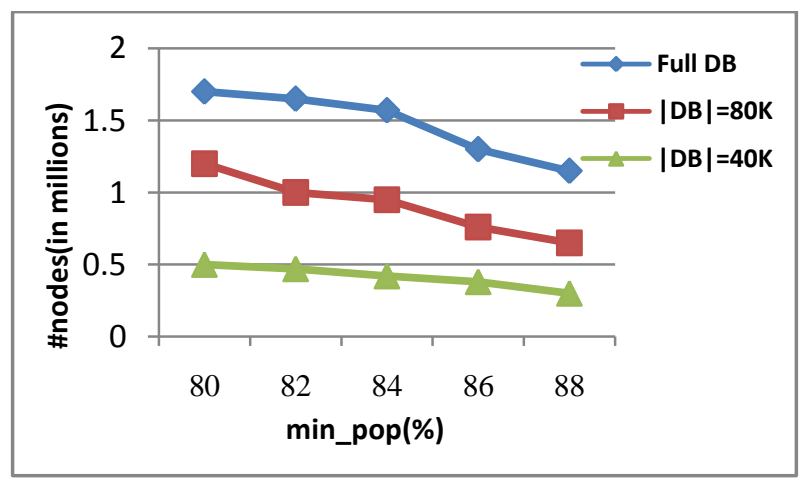

Fig 3: Compactness of the PCrimespatial-tree

Whenever the min_pop value increases the size of the tree structure gradually reduced in Chicago database, Fig 3 . In the increase in the size of the database, the number of nodes gets increased in most of the datasets. All the programs are written in Java and run in windows environment and processor is 1.3 $\mathrm{GHz}$. The programs which describes the run time indicates the total execution time along with selecting interesting attributes from large number of attributes in the database i.e., query processing.

\section{CONCLUSION}

In this paper the proposed algorithm to mine popular crime patterns from spatial databases. The popular crime patterns with different popularity values in a crime database are found. The system proceeded with the construction of PCrimeSpatial-tree with the help of $\mathrm{H}$-table. Later find out the popular crime patterns from the constructed tree. After the construction of tree take the projected data so that it is easier to find out the patterns from the different crime incidents taken. So in this paper popular crime patterns are extracted from spatial databases by using PCrimeSpatial-tree and in future scope this can be extended in distributed environment.

\section{REFERENCES}

[1] H. Chen, W. Chung, J. Xu, G. Wang, Y. Qin, M. Chau, Crime data mining: A general framework and some examples, IEEE Computer Journal 37 (4) (2004) 50-56.

[2] A. Mohammad, J. Mohsen, E. Martin, G. Uwe, F. Richard, Crimewalker: A recommendation model for suspect investigation, in: Proc. fifth ACM conference on Recommender systems, ACM, 2011, pp. 1-8.

[3] N. G. Khan, V. Bhaga, Effective data mining approach for crime-terrorpattern detection using clustering algorithm technique, Engineering Research and Technology International Journal Vol 2 (4) (2013), pp. 2043-2048.

[4] P. Phillips, I. Lee, Mining co-distribution patterns for large crime datasets, Expert Systems with Applications International Journal 39 (14) (2012) 11556-11563.

[5] O. Isafiade, A. Bagula, Citisafe: Adaptive spatial pattern knowledge using fp-growth algorithm for crime situation recognition, in: Proc. IEEE International Conference on Ubiquitous Intelligence and Computing, IEEE, 2013, pp. 551-556. 
[6] D. Wang, W. Ding, H. Lo, T. Stepinski, J. Salazar, M. Morabito, Crime hotspot mapping using the crime related factors- a spatial data mining approach, Applied Intelligence Journal 39 (4) (2013) 772-781.

[7] Brown, D.E. The regional crime analysis program (RECAP): A frame work for mining data to catch criminals," in Proceedings of the IEEE International J.S. Yeh, S.C. Lin, "A New Data Structure for Asynchronous Periodic Pattern Mining", Proc. 3rd Int'l Conf. Ubiquitous Information Management and Communication, pp. 426-431, 2009.

[8] Animesh Tripathy, Subhalaxmi Das, Prashanta KumarPatra "An Intelligent approach for mining frequent patterns in spatial data base systems using SQL" IEEE 2012

[9] J. Han, W. Gong, Y. Yin, "Mining Segment-Wise Periodic Patterns in Time Related Databases," Proc. ACM Int'l Conf. Knowledge Discovery and Data Mining, pp. 214-218, 1998.
[10] J. Yang, W. Wang, and P. S. Yu, "Mining Asynchronous Periodic Patterns in Time Series Data", IEEE Trans. on Knowledge and Data Engineering, Vol. 15, Issue 3, pp. 613-628, 2003

[11] K. Y. Huang, C.H. Chang, "SMCA: A General Model for Mining Asynchronous Periodic Patterns in Temporal Databases", IEEE Trans. On Knowledge and Data Engineering, Vol. 17, Issue 6, pp. 774-785, Jun, 2005.

[12] De Bruin, J.S. , Cocx, T.K. , Kosters, W.A. , Laros, J. And Kok, J.N. Data mining approaches to criminal career analysis," in Proceedings of the Sixth International Conference on Data Mining (ICDM ${ }^{\text {ec }} 06$ ), Pp. 171-177 (2006).

[13] Abraham, T. and de Vel, O. Investigative profiling with computer forensic log data and association rules," in Proceedings of the IEEE International Conference on Data Mining (ICDM'02), Pp. 11 - 18 (2006).

[14] BVS Varma, V. Valli Kumari.: Mining Popular Crime Patterns from Crime Datasets, Vol 4, Issue 5, October 2015, pp. $741-748$ 